\title{
Correlation between serum homocysteine, Galectin-3 concentration and atrial structural remodeling in atrial fibrillation patients
}

https://doi.org/10.1515/pteridines-2020-0008

received January 19, 2020; accepted April 8, 2020.

\begin{abstract}
Objective To investigate the correlation between serum homocysteine (Hcy), Galectin-3 concentration and atrial structural remodeling in atrial fibrillation (AF) patients.
\end{abstract}

Methods Twenty-five patients with persistent atrial fibrillation (PeAF), 24 patients with paroxysmal atrial fibrillation $(\mathrm{PaAF})$ and 23 healthy controls were included in the present work. All subjects received an echocardiography examination. Serum concentration of Hcy and Galectin-3 were also examined by Enzyme Linked Immunosorbent Assay (ELISA).

ResultsEchocardiography examination demonstrated that there were significant differences for LAD $(p=0.002)$, LVEF ( $p=0.005)$ and LVAI ( $p=0.0001)$ between the control, PaAF and PeAF groups. However, LVSD and LVDD were not significantly different between the three groups $\left(\mathrm{p}_{\text {all }}>0.05\right)$. There was a significant positive correlation between LAVI and serum Hcy level in both PaAF $\left(r_{\text {pearson }}=0.49, p=0.016\right)$ and PeAF $\left(r_{\text {pearson }}=0.51, p=0.009\right)$ groups. The correlation between LAVI and serum Galectin-3 concentration was also statistically significant for PaAF $\left(\mathrm{r}_{\text {pearson }}=0.54\right.$, $\mathrm{p}=0.006)$ and PeAF $\left(\mathrm{r}_{\text {pearson }}=0.60, \mathrm{p}=0.001\right)$ groups. Using serum Hcy as reference, diagnostic sensitivity and specificity were calculated as 72.00 (95\%CI: 50.61-87.93) and 62.50 (95\%CI: 40.59-81.20), respectively, with an AUC of 0.68 for PaAF and PeAF. For serum Galectin-3, the

*Corresponding author: Xuhui Sun, Department of Cardiology, $1^{\text {ST }}$ People's Hospital of Fuyang District Hanzhoug City, Zhejiang Province 311400 PR China, E-mail: sundotey@163.com Guiying Liang, Department of Cardiology, $1^{\text {ST }}$ People's Hospital of Fuyang District Hanzhoug City, Zhejiang Province 311400 PR China Xianchun Li, Department of electrocardiogram room, $1^{\text {sT }}$ People's Hospital of Fuyang District Hanzhoug City, Zhejiang Province 311400 PR China

Jian Yu, Department of Medicine, Hangzhou Fuyang Hospital of Traditional Chinese Medicine, Hanzhoug City, Zhejiang Province 311499 PR China sensitivity and specificity values were 64.00 (95\%CI:42.52$82.03)$ and 66.67 (95\%CI:44.68-84.37), respectively, with an AUC of 0.68 .

Conclusion: Serum Hcy and Galectin-3 were elevated in AF patients and thus may be potential markers of atrial structural remodeling. However, the diagnostic efficacy of PeAF from PaAF was limited by low AUC values.

Keywords: Hcy; Galectin-3; atrial structural remodeling; atrial fibrillation; serological marker.

\section{Introduction}

Atrial fibrillation (AF) is one of the most common arrhythmias seen in clinical practice. An epidemiological study reports the total incidence of AF in China to be $1.1 \%$ [1]. As the proportion of older persons in a population continues to increase, so does the incidence of atrial fibrillation. AF can lead to hemodynamic changes, left atrial thrombosis, and increased risk of stroke and heart failure. More generally, it is associated with a high rate of death or disability. In recent years, basic research on atrial fibrillation has made encouraging progress, and novel treatment methods (such as radiofrequency catheter ablation) have also improved the prognoses of AF patients. However, the recurrence rate after traditional drug therapy and radiofrequency catheter ablation remains high, especially in patients with persistent atrial fibrillation. Despite the great harm caused by AF, treatment options remain limited; therefore, it is of great significance to identify the mechanism of occurrence, development and maintenance of $\mathrm{AF}$.

At present, the pathogenesis of $\mathrm{AF}$ is not clear [2, 3]. Atrial remodeling [4], inflammatory response and oxidative stress [5-7] are considered to be important pathophysiological mechanisms of AF. Atrial structural remodeling is characterized by atrial fibrosis, dissolution of atrial myofibrils, apoptosis of atrial myocytes, increased 
or over-accumulated collagen in the extracellular matrix (ECM), obvious increase in collagen content and changes to components. Ultimately, this leads to uneven atrial conduction, further promoting atrial electrical remodeling and providing a pathological basis for the occurrence and development of atrial fibrillation $[8,9]$.

Hcy is an amino acid containing a sulfhydryl group; it mainly participates in the oxidative stress reaction in vivo and is a marker of oxidative stress in vivo [10,11]. It produces a large number of oxygen free radicals and peroxides in the process of oxidation-reduction in vivo. Active oxygen then directly affects calmodulin to cause intracellular calcium overload, change atrial ion channels and promote atrial electrical remodeling $[12,13]$. Galectin-3 is a member of the galectin family and can be expressed in many organs, such as the small intestine, spleen, colon and kidney, as well as in inflammatory cells such as mast cells [14], neutrophils and macrophages [15]. Galectin-3 is involved in a variety of pathophysiological processes, including inflammatory response and fibrosis [16], both considered to be key mechanisms and important markers of atrial and ventricular remodeling and heart failure [17-19]. However, the relationship between serum Hcy, galectin-3 levels and atrial structural remodeling in patients with atrial fibrillation remains unclear. In this study, we investigated the expression of Hcy and galectin-3 in serum of patients with atrial fibrillation and assessed the relationship with atrial structural remodeling.

\section{Material and methods}

\section{Patients}

Twenty-five patients with persistent atrial fibrillation (PeAF), 24 patients with paroxysmal atrial fibrillation $(\mathrm{PaAF})$ and 23 healthy controls were included in the present work. The subjects in the AF group had at least one 24-hour ambulatory ECG or had a clear history of atrial fibrillation confirmed by ECG. AF diagnosis was performed in accordance with the European guidelines for the management of atrial fibrillation [20-22]: 1) the absolute RR interval of ECG is different; 2) no obvious $\mathrm{P}$ wave can be seen on ECG; 3) the interval between the two atrial electrical activities is usually variable, and the time limit is generally less than $200 \mathrm{~ms}$.

Ethical approval: The research related to human use has been complied with all the relevant national regulations, institutional policies and in accordance the tenets of the Helsinki Declaration, and has been approved by the Ethical Committee of $1^{\text {ST }}$ People's Hospital of Fuyang District Hanzhoug City, Zhejiang Province 311400 PR China.

Informed consent: Informed consent has been obtained from all individuals included in this study

\section{Echocardiography examination}

All subjects were examined with echocardiography performed by experienced doctors in the ultrasound department of our hospital. Left atrial diameter (LAD), ascending aortic diameter (AO), right ventricular diameter, left ventricular posterior wall thickness (LVPWT), interventricular septal thickness (IVST), left ventricular end diastolic diameter (LVDD), left ventricular end systolic diameter (LVSD), pulmonary artery diameter and right and left atrial diameter were all recorded. The left ventricular ejection fraction (LVEF) was measured by the Simpson method.

\section{Measurement and calculation of left atrial volume}

Before measuring, subjects rested for 15 minutes. After minutes of calm breathing, probes were placed about $1-2 \mathrm{~cm}$ from the inner side of the apical beat to show the apical four chamber section toward the right shoulder. The image was frozen, and the left atrium volume was outlined and recorded as A1. The distance from the top of the left atrium to the midpoint of the line between the mitral annulus was measured and recorded as L1. The probe was rotated $90^{\circ}$ anticlockwise to display the apical two chamber section. The image was frozen, and the left atrium area was outlined and recorded as A2. The distance from the top of the left atrium to the midpoint of the line between the mitral annulus was measured and recorded as L2. The smaller value between L1 and L2 was selected and recorded as L. All the above measurements were continuously recorded for three cardiac cycles, and the average value was taken, Figure 1. The left atrial volume was calculated according to the formula: 8 (A1) (A2) / $3 \pi$ (L) and LAVI was calculated by: LAVI=LAV/BSA (ml/m2).

\section{Serum Hcy, Galectin-3 examination}

After patients had fasted for eight hours, $5 \mathrm{ml}$ of peripheral venous blood was collected from common vacuum vessels in the morning. After two hours of standing at room 

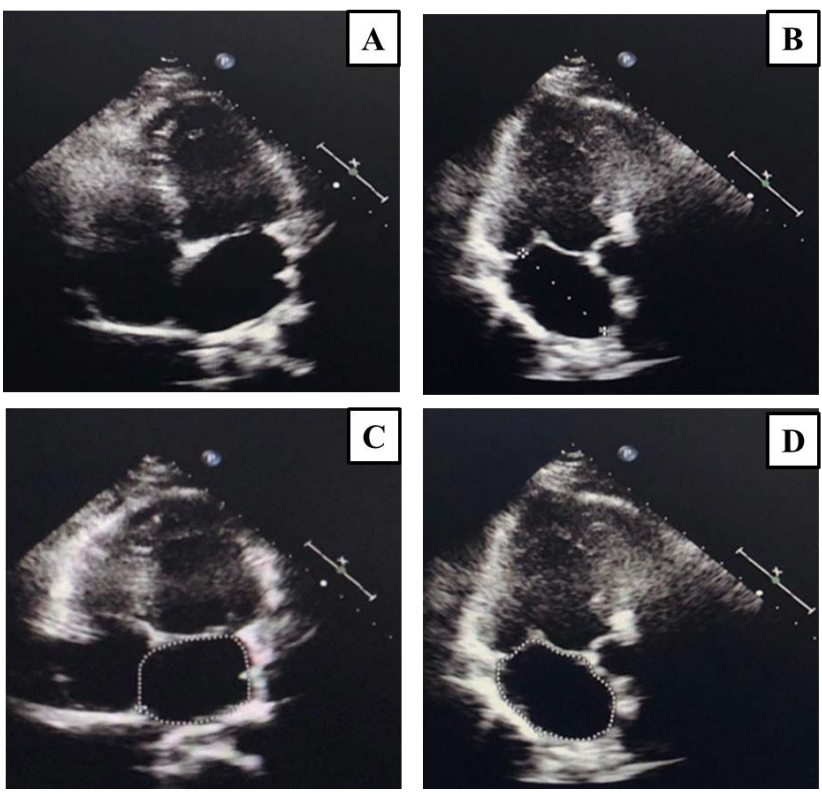

Figure 1: Measurement and calculation of left atrial volume(A: the distance from the top of the left atrium to the midpoint of the line between the mitral annulus; $\mathrm{B}$ : $\mathrm{t}$ he distance from the top of the left atrium to the midpoint of the line between the mitral annulus; C: outline the left atrium volume corresponding to L1; D outline the area of the left atrium corresponding to L2:).

temperature, the blood was centrifuged at $4000 \mathrm{r} / \mathrm{min}$ for 15 minutes. The upper serum was transferred to an EP tube and stored at - 80国 until use. Serum Hcy and Galectin-3 level was detected using an ELISA assay according to the manufacturer's instructions.

\section{Statistical analysis}

Statistical analysis was performed using STATA11.0 statistical software (http://www.stata.com). Measurement data were expressed with $\bar{x} \pm s$, and the comparison between groups was made based on the AVOVA test of the sample mean. Enumeration data were expressed with a relative number, and the comparison between groups was made based on the $\mathrm{c}^{2}$ test. The correlation was evaluated by Pearson correlation test. Regarding the diagnosis test, sensitivity, specificity, and the area under the ROC curve were calculated according to Bayes' theorem. $\mathrm{P}<0.05$ indicates statistical significance.

\section{Results \\ General characteristics of the included subjects}

General characteristics of the three subject groups are shown in Table 1. There were significant statistical differences between the three groups with respect to age $(p<0.05)$ and hypertension $(p<0.05)$. However, gender, body weight, height, diabetes, serum Scr, TC and TG were not statistical different between these groups $\left(\mathrm{p}_{\text {all }}>0.05\right)$.

\section{Echocardiography}

Echocardiography examination revealed significant differences in LAD ( $p=0.002)$, LVEF $(p=0.005)$ and LVAI $(\mathrm{p}=0.0001)$ between the control, PaAF and PeAF groups. However, LVSD and LVDD were not significantly different across the groups ( $\left.\mathrm{p}_{\text {all }}>0.05\right)$, Table 2.

\section{Serum Hcy, Galectin-3 concentration}

The serum Hcy concentrations of control, PaAF and PeAF groups were $8.43 \pm 2.79(\mu \mathrm{mol} / \mathrm{L}), 10.91 \pm 4.28(\mu \mathrm{mol} / \mathrm{L})$, and $14.08 \pm 5.00(\mu \mathrm{mol} / \mathrm{L})$, respectively. For serum Galectin-3, concentration values were $1.44 \pm 0.18(\mathrm{ng} / \mathrm{ml}), 1.59 \pm 0.26(\mathrm{ng} /$ $\mathrm{ml})$ and $1.81 \pm 0.33(\mathrm{ng} / \mathrm{ml})$ for the control, PaAF and PeAF groups, respectively, Table $\mathbf{3}$. There is a statistically significant difference in serum Hcy and Galectin-3 concentration between control, PaAF and PeAF groups, Figure 2.

\section{Correlation between serum Hcy Galectin-3 concentration and LAVI}

The correlations between serum Hcy, Galectin-3 concentration and LAVI are shown in Table 4. There were a significant positive correlation between LAVI and serum Hcy level in PaAF ( $\left.\mathrm{r}_{\text {pearson }}=0.49, \mathrm{p}=0.016\right)$ and PeAF $\left(r_{\text {pearson }}=0.51, p=0.009\right)$ groups. The correlation between LAVI and serum Galectin-3 concentration was also statistically significant for PaAF ( $\mathrm{r}_{\text {pearson }}=0.54, \mathrm{p}=0.006$ ) and PeAF $\left(\mathrm{r}_{\text {pearson }}=0.60, \mathrm{p}=0.001\right)$ groups, Figure 3 . 
Table 1: The main characteristics of the included subjects.

\begin{tabular}{|c|c|c|c|c|c|}
\hline Characteristics & Control(n=23) & $\operatorname{PaAF}(n=24)$ & $\operatorname{PeAF}(n=25)$ & F/chi-square & p-value \\
\hline Age(year) & $57.2 \pm 9.7$ & $62.4 \pm 10.8$ & $66.9 \pm 8.7$ & 5.92 & 0.004 \\
\hline Gender(n,\%) & & & & 0.07 & 0.79 \\
\hline Male & 11 & 11 & 11 & & \\
\hline Female & 12 & 13 & 14 & & \\
\hline Weight(kg) & $63.7 \pm 10.5$ & $61.4 \pm 9.8$ & $59.5 \pm 10.6$ & 0.99 & 0.37 \\
\hline Height(cm) & $166.7 \pm 8.2$ & $164.2 \pm 7.9$ & $163.5 \pm 9.2$ & & \\
\hline Hypertension(n,\%) & & & & 7.64 & 0.006 \\
\hline Yes & 3 & 11 & 13 & & \\
\hline No & 20 & 13 & 12 & & \\
\hline Diabetes(n,\%) & & & & 0.15 & 0.69 \\
\hline Yes & 2 & 2 & 3 & & \\
\hline No & 21 & 22 & 22 & & \\
\hline $\operatorname{Scr}(\mu \mathrm{mol} / \mathrm{L})$ & $61.4 \pm 18.3$ & $66.2 \pm 22.7$ & $64.9 \pm 24.1$ & 0.30 & 0.74 \\
\hline $\mathrm{TC}(\mathrm{mmol} / \mathrm{L})$ & $4.42 \pm 1.23$ & $4.56 \pm 1.04$ & $4.24 \pm 1.43$ & 0.41 & 0.67 \\
\hline $\mathrm{TG}(\mathrm{mmol} / \mathrm{L})$ & $1.33 \pm 0.65$ & $1.56 \pm 0.68$ & $1.49 \pm 0.72$ & 0.69 & 0.50 \\
\hline
\end{tabular}

PeAF=Persistent atrial fibrillation

$\mathrm{PaAF}=$ Paroxysmal atrial fibrillation

$\mathrm{TC}=$ total cholesterol

$\mathrm{TG}=$ total glyceride

Table 2: The main measurement values of echocardiography for the 3 groups.

\begin{tabular}{llllll}
\hline Echocardiography & Control & PaAF & PeAF & F & p-value \\
\hline LAD $(\mathrm{mm})$ & $33.2 \pm 4.8$ & $35.9 \pm 6.8$ & $40.7 \pm 9.2$ & 6.68 & 0.002 \\
LVSD $(\mathrm{mm})$ & $31.2 \pm 4.2$ & $31.8 \pm 5.1$ & $32.9 \pm 5.1$ & 0.77 & 0.47 \\
$\operatorname{LVDD}(\mathrm{mm})$ & $45.6 \pm 6.2$ & $44.8 \pm 7.6$ & $46.3 \pm 8.4$ & 0.25 & 0.78 \\
$\operatorname{LVEF}(\%)$ & $65.3 \pm 4.8$ & $60.3 \pm 9.1$ & $57.8 \pm 8.7$ & 5.65 & 0.005 \\
LAVI $(\mathrm{ml} / \mathrm{m} 2)$ & $30.96 \pm 4.73$ & $35.30 \pm 7.61$ & $39.76 \pm 7.53$ & 10.07 & 0.0001 \\
\hline
\end{tabular}

LAD :left atrial diameter

LVSD :left ventricular end systolic diameter

LVDD :left ventricular end diastolic diameter

LVEF :left ventricular ejection fraction

LAVI :left atrial volume index

Table 3: Serum Hcy, Galectin-3 concentration for the 3 groups.

\begin{tabular}{llllll}
\hline Characteristics & Control & PaAF & PeAF & F & p-value \\
\hline Hcy $(\mu \mathrm{mol} / \mathrm{L})$ & $8.43 \pm 2.79$ & $10.91 \pm 4.28$ & $14.08 \pm 5.00$ & 5.91 & 0.004 \\
Galectin-3 $(\mathrm{ng} / \mathrm{ml})$ & $1.44 \pm 0.18$ & $1.59 \pm 0.26$ & $1.81 \pm 0.33$ & 11.66 & $<0.0001$ \\
\hline
\end{tabular}



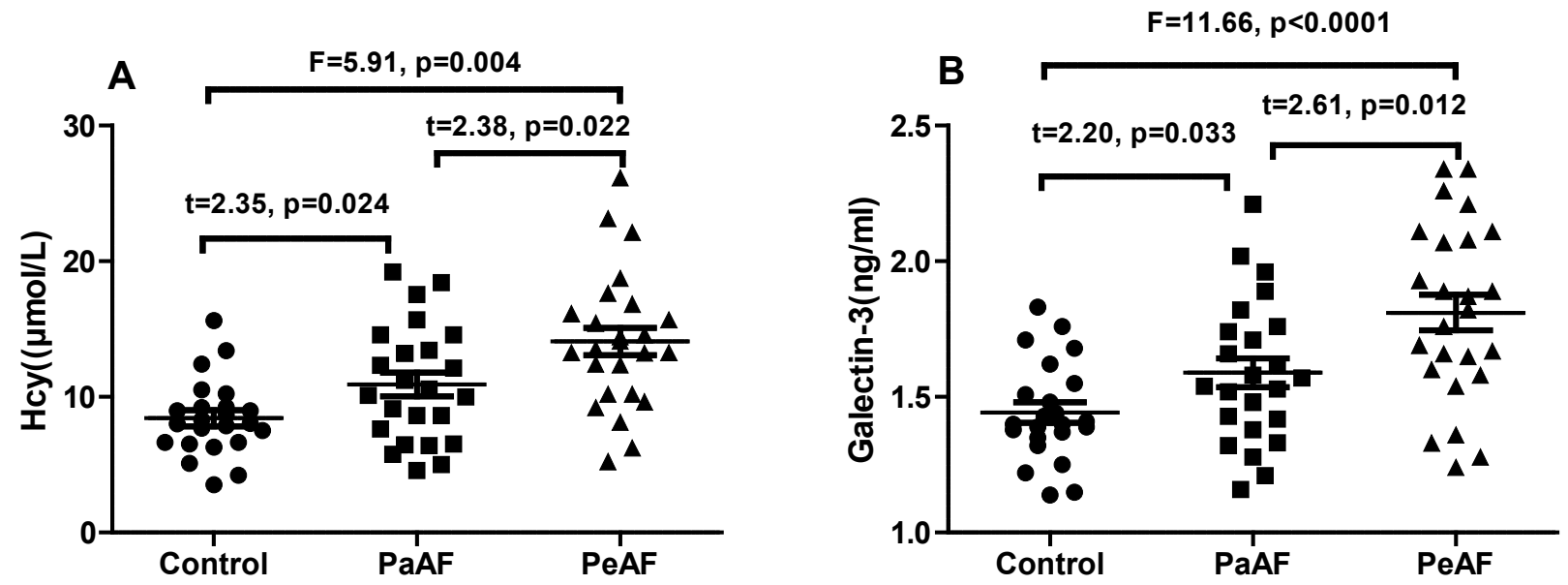

Figure 2: Scater plot of serum Hcy, Galectin-3 concentration for different groups(A: Serum Hcy for control, PaAF, PeAF groups; B: Serum Galectin-3 for control, PaAF, PeAF groups).

A
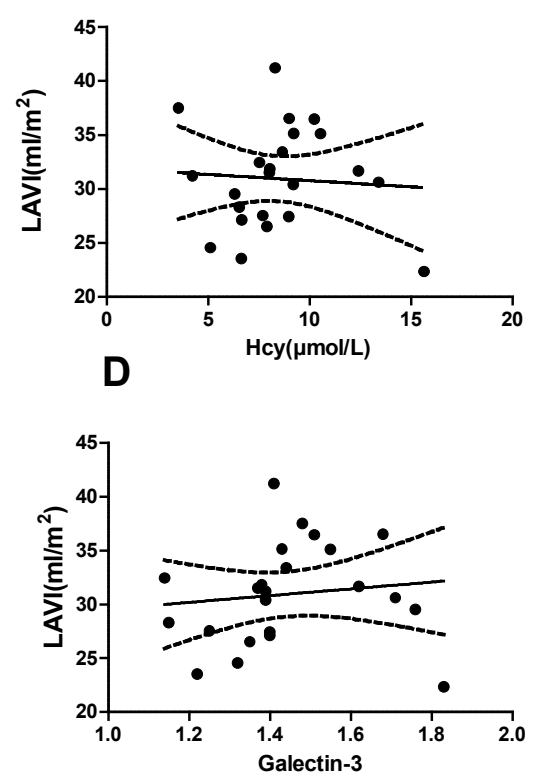

B
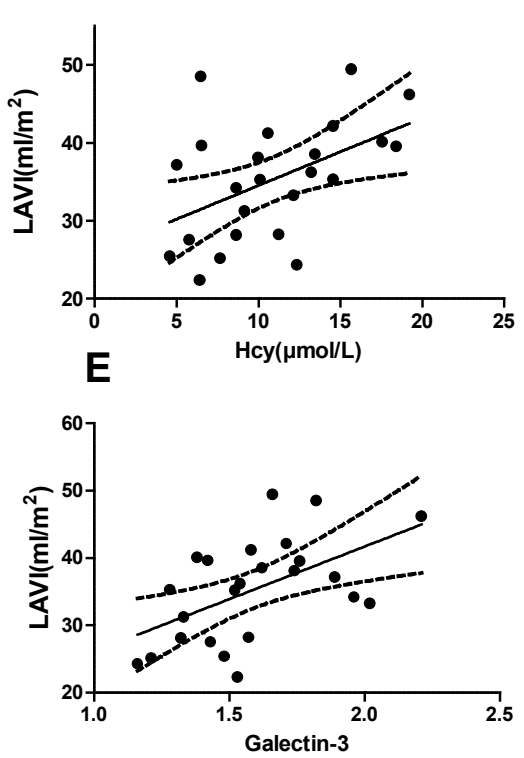

C

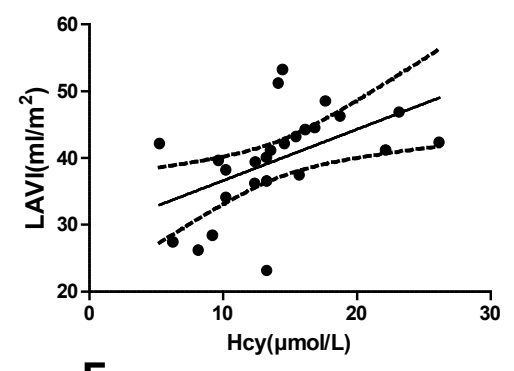

$\mathbf{F}$

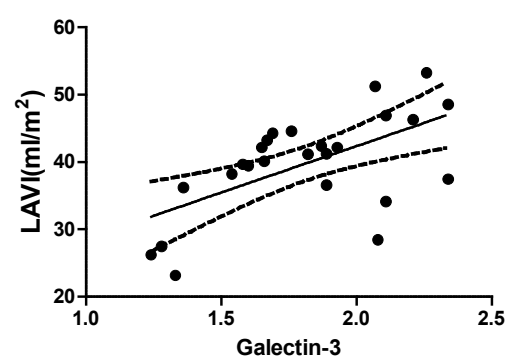

Figure 3: The correlation between serum Hcy, Galectin-3 concentration and LAVI in different groups(A: Correlation between LAVI and Hcy in control group; B: Correlation between LAVI and Hcy in PaAF group; C: Correlation between LAVI and Hcy in PeAF group; D: Correlation between LAVI and Galectin-3 in control group; E:Correlation between LAVI and Galectin-3 in PaAF group; Correlation between LAVI and Galectin-3 in PeAF group).

\section{Serum Hcy and Galectin-3 as biomarker for diagnosis of PeAF}

Using serum Hcy as reference, diagnostic sensitivity and specificity values were determined to be 72.00 (95\%CI:50.61-87.93) and 62.50 (95\%CI:40.59-81.20), respectively, with an AUC of 0.68 between PaAF and PeAF.
For serum Galectin-3, the sensitivity and specificity values were 64.00 (42.52-82.03) and 66.67 (95\%CI:44.68-84.37), respectively (Table 5), with an AUC of 0.68, Figure 4. 
Table 4: Correlation between serum Hcy, Galectin-3 concentration and LAVI.

\begin{tabular}{|c|c|c|c|c|}
\hline Correlation & $r_{\text {pearson }}$ & $95 \% \mathrm{Cl}$ & R square & $\mathbf{p}$ \\
\hline \multicolumn{5}{|l|}{ LVAl vs Hcy } \\
\hline Control & -0.067 & -0.47 to 0.36 & 0.005 & 0.76 \\
\hline PaAF & 0.49 & 0.10 to 0.74 & 0.24 & 0.016 \\
\hline PeAF & 0.51 & 0.14 to 0.75 & 0.26 & 0.009 \\
\hline \multicolumn{5}{|c|}{ LAVI vs Galectin-3 } \\
\hline Control & 0.21 & -0.31 to 0.51 & 0.014 & 0.58 \\
\hline $\mathrm{PaAF}$ & 0.54 & 0.18 to 1.78 & 0.29 & 0.006 \\
\hline PeAF & 0.60 & 0.27 to 0.80 & 0.36 & 0.001 \\
\hline
\end{tabular}

Table 5: The diagnostic efficacy of serum Hcy and Galectin-3 as biomarker for diagnosis of PeAF.

\begin{tabular}{lllllll}
\hline Reference & Sen(\%) & Sep(\%) & AUC $(95 \% \mathrm{Cl})$ & LR & p & Cut off \\
\hline Hcy & $72.00(50.61-87.93)$ & $62.50(40.59-81.20)$ & $0.68(0.54-0.84)$ & 2.16 & 0.023 & 12.24 \\
Galectin-3 & $64.00(42.52-82.03)$ & $66.67(44.68-84.37)$ & $0.71(0.56-0.85)$ & 1.92 & 0.013 & 1.67 \\
\hline
\end{tabular}

A

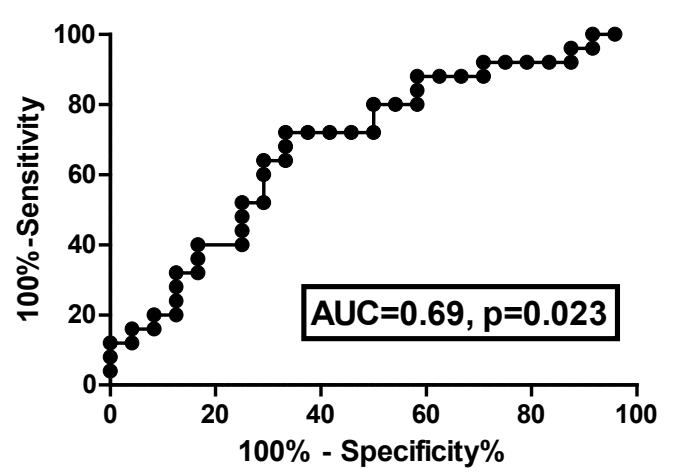

B

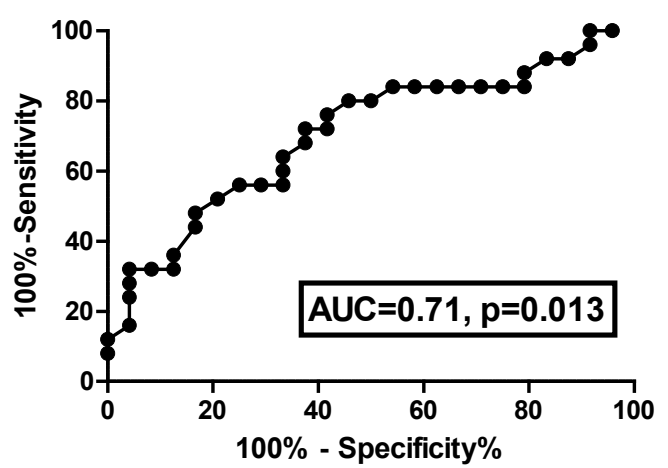

Figure 4: Roc curve for serum Hcy and Galectin-3 as biomarker for diagnosis of PeAF(A:serum Hcy in diagnosis of PeAF; B: serum Galectin-3 in diagnosis of PeAF).

\section{Discussion}

In the present work, we found that LAD ( $\mathrm{p}=0.002)$, LVEF $(\mathrm{p}=0.005)$ and LVAI $(\mathrm{p}=0.0001)$ are significantly higher in the AF group in comparison to controls. This indicates atrial structural remodelling in $\mathrm{AF}$ patients. The results also indicate that LAVI is positively correlated with both serum Hcy and Galectin-3 in AF patients. This may be evidence that Hcy and Galectin-3 acts as import serological markers for atrial structural remodeling. Therefore, as serum Hcy and Galectin-3 are elevated in AF patients, they may represent a potential marker of atrial structural remodeling.. However, the diagnostic efficacy of PeAF from PaAF was limited by low AUC.

Mihm and colleagues [23] found that oxidative damage in human AF alters myofibrillar energetics and contributes to atrial contractile dysfunction. Kim et al. [24] also found that expression of both monoamine oxidase $B$ and tyrosinase related protein-1 is up-regulated in the right auricles of patients with atrial fibrillation; furthermore, expression of the antioxidant gene glutathione peroxidase-1 is down regulated, while active oxygen in 
vivo isup regulated. Lin and colleagues [25] found that evidence of oxidative injury and deletion of mtDNA in cardiac muscle is increased in the patients with AF, which may impair of bioenergetic function of mitochondria and ultimately induce the oxidative vicious oxidative cycle involved in the pathogenesis of atrial myopathy in AF. The aforementioned reorts suggest that oxidative stress plays a role in the process of AF. Hcy an amino acid containing a sulfhydryl group, and is thus able to participate in various important redox reactions in vivo, in addition to being a marker of oxidative stress in vivo. Hcy produces a large number of oxygen free radicals and peroxides by its own oxidation [26]. This active oxygen directly affects calmodulin, causing intracellular calcium overload and inducuing changes to atrial ion channels that ultimately lead to atrial electrical remodeling and promote the occurrence of atrial fibrillation. In a large prospective study, Cai et al. [27] found that high Hcy level is a risk factor for the occurrence of atrial fibrillation, potentially due to the fact that long-term high Hcy levels can interfere with the potassium channel currents of atrial myocytes and cause electrical remodeling of the atrium. Nasso et al. [28] found that Hcy levels in peripheral blood are an independent predictor of recurrence in patients with atrial fibrillation (including paroxysmal and persistent atrial fibrillation) after epicardial ablation.

However, the model that oxidative stress mediates the pathological consequences associated with elevated homocysteine remains controversial [29]. For example, lowering elevated homocysteine levels does not decrease pathological consequences associated with elevated homocysteine [30]. Therefore, the relationship between oxidative stress, hyperhomocysteinemia and the pathological consequences of heart disease requires further exploration. Hcy is an intermediate metabolite in the metabolic pathwayd of cysteine and methionine. It may undergo remethylation to methionine in a reaction catalyzed by ethylenetetrahydrofolate homocysteine methyltransferase [29]. Hcy also can be recognized and activated by methionyl-tRNA synthetase to produce Hcythiolactone, which can react with the $\varepsilon$-amino group of a protein lysine residue. The N-hcy-linked protein carrying a free thiol group can influence protein structure and function, thus leading to severe diseases [31].

Goktekin [32] selected 52 patients with nonvalvular atrial fibrillation and 33 age- and gender-matched control participants for his study, in which he detected markers of fibrosis and inflammation in their serum, including galectin-3, matrix metalloproteinase-9, MMP-9, apolipoprotein-2, Lcn2 and type III procollagen $\mathrm{N}$-terminal protein of type III proco. The results showed that levels of galectin-3, MMP-9, PIIINP, NLR and hs CRP were higher in the $\mathrm{AF}$ group than in the control group. Addiiotnally, levels of galectin-3, MMP-9, PIIINP and NLR were positively correlated with left atrial volume index.

Our work is consistent with these findings. Moreover, we also determined that galectin-3 is not only related to the occurrence of AF, but also implicated in its development and maintenance. Yalcin et al. [33] found that serum galectin-3 levels in patients with paroxysmal atrial fibrillation with left ventricular ejection fraction retention were higher than those of the control group, and galectin-3 levels were positively correlated with the degree of atrial fibrosis shown by 3D delayed enhanced MR imaging. Gurses et al. [34] found that the level of serum galectin-3 in patients with $\mathrm{AF}$ with preserved ejection fraction was higher than that of the age-matched control group, in addition to being positively correlated with left atrial volume index. Both of the above studies indicate that galectin-3 is related to left atrial fibrosis, participates in the process of left atrial structural remodeling and promotes the development of atrial fibrillation.

In conclusion, serum Hcy and Galectin-3 are elevated in $\mathrm{AF}$ patients and may be a potential marker for atrial structural remodeling. However, due to low AUC, the diagnostic efficacy of PeAF from PaAF is currently limited.

\section{References}

1. Xing L, Lin M, Du Z, Jing L, Tian Y, Yan H, Ren G, Dong Y, Sun Q, Dai D, Shi L, Chen H, Liu S. Epidemiology of atrial fibrillation in northeast China: a cross-sectional study, 2017-2019. Heart 2019.

2. Ciconte G, Conti M, Evangelista M, Pappone C. Atrial Fibrillation in Autoimmune Rheumatic Diseases: from Pathogenesis to Treatment. Rev Recent Clin Trials 2018;13:170-175.

3. Hu YF, Chen YJ, Lin YJ, Chen SA. Inflammation and the pathogenesis of atrial fibrillation. Nat Rev Cardiol 2015;12:23043.

4. Mulder MJ, Kemme M, Visser CL, Hopman L, van Diemen PA, van de Ven PM, Götte M, Danad I, Knaapen P, van Rossum AC, Allaart CP. Left atrial sphericity as a marker of atrial remodeling: Comparison of atrial fibrillation patients and controls. Int J Cardiol 2020;304:69-74.

5. Pezhouman A, Cao H, Fishbein MC, Belardinelli L, Weiss JN, Karagueuzian HS. Atrial Fibrillation Initiated by Early Afterdepolarization-Mediated Triggered Activity during Acute Oxidative Stress: Efficacy of Late Sodium Current Blockade. J Heart Health 2018;4.

6. Korantzopoulos P, Letsas K, Fragakis N, Tse G, Liu T. Oxidative stress and atrial fibrillation: an update. Free Radic Res 2018;52:1199-1209.

7. Karam BS, Chavez-Moreno A, Koh W, Akar JG, Akar FG. Oxidative stress and inflammation as central mediators of 
atrial fibrillation in obesity and diabetes. Cardiovasc Diabetol 2017;16:120.

8. Sohns C, Marrouche NF. Atrial fibrillation and cardiac fibrosis. Eur Heart J 2019.

9. Lau CP, Tse HF, Siu CW, Gbadebo D. Atrial electrical and structural remodeling: implications for racial differences in atrial fibrillation. J Cardiovasc Electrophysiol 2012;23 Suppl 1:S36-40.

10. Bourgonje AR, Abdulle AE, Al-Rawas AM, Al-Maqbali M, Al-Saleh M, Enriquez MB, Al-Siyabi S, Al-Hashmi K, Al-Lawati I, Bulthuis M, Mulder DJ, Gordijn SJ, van Goor H, Saleh J. Systemic Oxidative Stress Is Increased in Postmenopausal Women and Independently Associates with Homocysteine Levels. Int J Mol Sci 2020;21.

11. Feng PN, Liang YR, Lin WB, Yao ZR, Chen DB, Chen PS, Ouyang J. Homocysteine induced oxidative stress in human umbilical vein endothelial cells via regulating methylation of SORBS1. Eur Rev Med Pharmacol Sci 2018;22:6948-6958.

12. Maille B, Das M, Hussein A, Shaw M, Chaturvedi V, Williams E, Morgan M, Ronayne C, Snowdon RL, Gupta D. Reverse electrical and structural remodeling of the left atrium occurs early after pulmonary vein isolation for persistent atrial fibrillation. J Interv Card Electrophysiol 2019.

13. Adeniran I, Maclver DH, Garratt CJ, Ye J, Hancox JC, Zhang H. Effects of Persistent Atrial Fibrillation-Induced Electrical Remodeling on Atrial Electro-Mechanics - Insights from a 3D Model of the Human Atria. PLoS One 2015;10:e0142397.

14. Chen HY, Sharma BB, Yu L, Zuberi R, Weng IC, Kawakami Y, Kawakami T, Hsu DK, Liu FT. Role of galectin-3 in mast cell functions: galectin-3-deficient mast cells exhibit impaired mediator release and defective JNK expression. J Immunol 2006;177:4991-7.

15. Quenum Zangbede FO, Chauhan A, Sharma J, Mishra BB. Galectin-3 in M2 Macrophages Plays a Protective Role in Resolution of Neuropathology in Brain Parasitic Infection by Regulating Neutrophil Turnover. J Neurosci 2018;38:6737-6750.

16. Liu FT, Hsu DK. The role of galectin-3 in promotion of the inflammatory response. Drug News Perspect 2007;20:455-60.

17. Zhang G, Wu Y. Circulating Galectin-3 and Atrial Fibrillation Recurrence after Catheter Ablation: A Meta-Analysis. Cardiovasc Ther 2019;2019:4148129.

18. Clementy N, Piver E, Bisson A, Andre C, Bernard A, Pierre B, Fauchier L, Babuty D. Galectin-3 in Atrial Fibrillation: Mechanisms and Therapeutic Implications. Int J Mol Sci 2018;19.

19. Lippi G, Cervellin G, Sanchis-Gomar F. Galectin-3 in atrial fibrillation: Simple bystander, player or both. Clin Biochem 2015;48:818-22.

20. Camm AJ, Lip GY, De Caterina R, Savelieva I, Atar D, Hohnloser SH, Hindricks G, Kirchhof P. 2012 focused update of the ESC Guidelines for the management of atrial fibrillation: an update of the 2010 ESC Guidelines for the management of atrial fibrillation--developed with the special contribution of the European Heart Rhythm Association. Europace 2012;14:1385413.

21. Camm AJ, Lip GY, De Caterina R, Savelieva I, Atar D, Hohnloser SH, Hindricks G, Kirchhof P. 2012 focused update of the ESC Guidelines for the management of atrial fibrillation: an update of the 2010 ESC Guidelines for the management of atrial fibrillation. Developed with the special contribution of the
European Heart Rhythm Association. Eur Heart J 2012;33:2719 47.

22. Savelieva I, Kakouros N, Kourliouros A, Camm AJ. Upstream therapies for management of atrial fibrillation: review of clinical evidence and implications for European Society of Cardiology guidelines. Part II: secondary prevention. Europace 2011;13:610-25.

23. Mihm MJ, Yu F, Carnes CA, Reiser PJ, McCarthy PM, Van Wagoner DR, Bauer JA. Impaired myofibrillar energetics and oxidative injury during human atrial fibrillation. Circulation 2001;104:174-80.

24. Kim YH, Lim DS, Lee JH, Lim DS, Shim WJ, Ro YM, Park GH, Becker KG, Cho-Chung YS, Kim MK. Gene expression profiling of oxidative stress on atrial fibrillation in humans. Exp Mol Med 2003;35:336-49.

25. Lin PH, Lee SH, Su CP, Wei YH. Oxidative damage to mitochondrial DNA in atrial muscle of patients with atrial fibrillation. Free Radic Biol Med 2003;35:1310-8.

26. Guéant Rodriguez RM, Spada R, Pooya S, Jeannesson E, Moreno Garcia MA, Anello G, Bosco P, Elia M, Romano A, Alberto JM, Juillière $Y$, Guéant JL. Homocysteine predicts increased NT-pro-BNP through impaired fatty acid oxidation. Int J Cardiol 2013;167:768-75.

27. Cai BZ, Gong DM, Liu Y, Pan ZW, Xu CQ, Bai YL, Qiao GF, Lu YJ, Yang BF. Homocysteine inhibits potassium channels in human atrial myocytes. Clin Exp Pharmacol Physiol 2007;34:851-5.

28. Nasso G, Bonifazi R, Romano V, Brigiani MS, Fiore F, Bartolomucci F, Lamarra M, Fattouch K, Rosano G, Gaudino M, Spirito R, Gaudio C, Speziale G. Increased plasma homocysteine predicts arrhythmia recurrence after minimally invasive epicardial ablation for nonvalvular atrial fibrillation. J Thorac Cardiovasc Surg 2013;146:848-53.

29. Loscalzo J. The oxidant stress of hyperhomocyst(e)inemia. J Clin Invest 1996;98:5-7.

30. Bønaa KH, Njølstad I, Ueland PM, Schirmer H, Tverdal A, Steigen T, Wang H, Nordrehaug JE, Arnesen E, Rasmussen $\mathrm{K}$, NORVIT Trial Investigators. Homocysteine lowering and cardiovascular events after acute myocardial infarction. N Engl J Med 2006;354:1578-88.

31. Xu L, Chen J, Gao J, Yu H, Yang P. Crosstalk of homocysteinylation, methylation and acetylation on histone H3. Analyst 2015;140:3057-63.

32. Sonmez O, Ertem FU, Vatankulu MA, Erdogan E, Tasal A, Kucukbuzcu S, Goktekin O. Novel fibro-inflammation markers in assessing left atrial remodeling in non-valvular atrial fibrillation. Med Sci Monit 2014;20:463-70.

33. Yalcin MU, Gurses KM, Kocyigit D, Canpinar H, Canpolat $\mathrm{U}$, Evranos B, Yorgun H, Sahiner ML, Kaya EB, Hazirolan T, Tokgozoglu L, Oto MA, Ozer N, Guc D, Aytemir K. The Association of Serum Galectin-3 Levels with Atrial Electrical and Structural Remodeling. J Cardiovasc Electrophysiol 2015;26:635-40.

34. Gurses KM, Yalcin MU, Kocyigit D, Canpinar H, Evranos B, Yorgun H, Sahiner ML, Kaya EB, Ozer N, Tokgozoglu L, Oto MA, Guc D, Aytemir K. Effects of persistent atrial fibrillation on serum galectin-3 levels. Am J Cardiol 2015;115:647-51. 